\title{
Blow-up criteria for 3D nematic liquid crystal models in a bounded domain
}

\author{
Jishan Fan' ${ }^{1}$ Gen Nakamura² and Yong Zhou ${ }^{3 *}$
}

\author{
"Correspondence: \\ yzhoumath@zjnu.edu.cn \\ ${ }^{3}$ Department of Mathematics, \\ Zhejiang Normal University, Jinhua, \\ P.R. China \\ Full list of author information is \\ available at the end of the article
}

\begin{abstract}
In this paper we prove some blow-up criteria for two 3D density-dependent nematic liquid crystal models in a bounded domain.
\end{abstract}

MSC: 35Q30; 76D03; 76D09

Keywords: liquid crystal; blow-up criterion; bounded domain

\section{Introduction}

Let $\Omega \subseteq \mathbb{R}^{3}$ be a bounded domain with smooth boundary $\partial \Omega$, and let $v$ be the unit outward normal vector on $\partial \Omega$. We consider the regularity criterion to the density-dependent incompressible nematic liquid crystal model as follows [1-4]:

$$
\begin{aligned}
& \operatorname{div} u=0, \\
& \partial_{t} \rho+\operatorname{div}(\rho u)=0, \\
& \partial_{t}(\rho u)+\operatorname{div}(\rho u \otimes u)+\nabla \pi-\Delta u=-\nabla \cdot(\nabla d \odot \nabla d), \\
& \partial_{t} d+u \cdot \nabla d+\left(|d|^{2}-1\right) d-\Delta d=0,
\end{aligned}
$$

in $(0, \infty) \times \Omega$ with initial and boundary conditions

$$
\begin{aligned}
& (\rho, u, d)(\cdot, 0)=\left(\rho_{0}, u_{0}, d_{0}\right) \quad \text { in } \Omega, \\
& u=0, \quad \partial_{\nu} d=0 \quad \text { on } \partial \Omega,
\end{aligned}
$$

where $\rho$ denotes the density, $u$ the velocity, $\pi$ the pressure, and $d$ represents the macroscopic molecular orientations, respectively. The symbol $\nabla d \odot \nabla d$ denotes a matrix whose $(i, j)$ th entry is $\partial_{i} d \partial_{j} d$, and it is easy to find that $\nabla d \odot \nabla d=\nabla d^{T} \nabla d$.

When $d$ is a given constant vector, then (1.1)-(1.3) represent the well-known densitydependent Navier-Stokes system, which has received many studies; see [5-7] and references therein.

When $\rho=1$, Guillén-González et al. [8] proved the blow-up criterion

$$
\int_{0}^{T}\left(\|u(t)\|_{L^{q}}^{\frac{2 q}{q-3}}+\|\nabla d(t)\|_{L^{q}}^{\frac{2 q}{q-3}}\right) d t<\infty \quad \text { with } 3<q \leq \infty
$$

and $0<T<\infty$.

@ 2013 Fan et al.; licensee Springer. This is an Open Access article distributed under the terms of the Creative Commons Attribution License (http://creativecommons.org/licenses/by/2.0), which permits unrestricted use, distribution, and reproduction in any medium, provided the original work is properly cited. 
It is easy to prove that the problem (1.1)-(1.6) has a unique local-in-time strong solution $[6,9]$, and thus we omit the details here. The aim of this paper is to consider the regularity criterion; we will prove the following theorem.

Theorem 1.1 Let $\rho_{0} \in W^{1, q}(\Omega), u_{0} \in H_{0}^{1}(\Omega) \cap H^{2}(\Omega), d_{0} \in H^{3}(\Omega)$ with $3<q \leq 6$ and $\rho_{0} \geq$ 0 , div $u_{0}=0$ in $\Omega$ and $\partial_{\nu} d_{0}=0$ on $\partial \Omega$. We also assume that the following compatibility condition holds true: $\exists\left(\nabla \pi_{0}, g\right) \in L^{2}(\Omega)$ such that

$$
\nabla \pi_{0}-\Delta u_{0}+\nabla \cdot\left(\nabla d_{0} \odot \nabla d_{0}\right)=\sqrt{\rho_{0}} g \quad \text { in } \Omega
$$

Let $(\rho, u, d)$ be a local strong solution to the problem (1.1)-(1.6). If u satisfies

$$
\int_{0}^{T}\|u(t)\|_{L^{q}}^{\frac{2 q}{q-3}} d t<\infty \quad \text { with } 3<q \leq \infty
$$

and $0<T<\infty$, then the solution $(\rho, u, d)$ can be extended beyond $T>0$.

Remark 1.1 When $\rho \equiv 1$, our result improves (1.7) to (1.8).

Remark 1.2 By similar calculations as those in [6], we can replace $L^{q}$-norm in (1.8) by $L_{w}^{q}$-norm, and thus we omit the details here.

Remark 1.3 When the space dimension $n=2$, we can prove that the problem (1.1)-(1.6) has a unique global-in-time strong solution by the same method as that in [10], and thus we omit the details here.

Next we consider another liquid model: (1.1), (1.2), (1.3), (1.5), (1.6) and

$$
\partial_{t} d+u \cdot \nabla d-\Delta d=|\nabla d|^{2} d
$$

with $|d| \equiv 1$ in $(0, \infty) \times \Omega$. Li and Wang [9] proved that the problem has a unique local strong solution. When $\Omega:=\mathbb{R}^{3}$, Fan et al. [11] proved a regularity criterion. The aim of this paper is to study the regularity criterion of the problem in a bounded domain. We will prove the following theorem.

Theorem 1.2 Let the initial data satisfy the same conditions in Theorem 1.1 and $\left|d_{0}\right| \equiv 1$ in $\Omega$. Let $(\rho, u, d)$ be a local strong solution to the problem (1.1)-(1.3), (1.5), (1.6) and (1.9). If $u$ and $\nabla d$ satisfy

$$
\int_{0}^{T}\left(\|u(t)\|_{L^{q}}^{\frac{2 q}{q-3}}+\|\nabla d\|_{L^{q}}^{\frac{2 q}{q-3}}\right) d t<\infty \quad \text { with } 3<q \leq \infty
$$

and $0<T<\infty$, then the solution $(\rho, u, d)$ can be extended beyond $T>0$.

\section{Proof of Theorem 1.1}

We only need to establish a priori estimates.

Below we shall use the notation

$$
\int=\int_{\Omega}
$$


First, thanks to the maximum principle, it follows from (1.1) and (1.2) that

$$
0 \leq \rho \leq\left\|\rho_{0}\right\|_{L^{\infty}}<\infty
$$

Testing (1.3) by $u$ and using (1.1) and (1.2), we see that

$$
\frac{1}{2} \frac{d}{d t} \int \rho u^{2} d x+\int|\nabla u|^{2} d x=-\int(u \cdot \nabla) d \cdot \Delta d d x .
$$

Testing (1.4) by $-\Delta d+\left(|d|^{2}-1\right) d$ and using (1.1), we find that

$$
\begin{aligned}
& \frac{d}{d t} \int\left(\frac{1}{2}|\nabla d|^{2}+\frac{1}{4}\left(|d|^{2}-1\right)^{2}\right) d x+\int\left(-\Delta d+\left(|d|^{2}-1\right) d\right)^{2} d x \\
& \quad=\int(u \cdot \nabla) d \cdot \Delta d d x .
\end{aligned}
$$

Summing up (2.2) and (2.3), we have the well-known energy inequality

$$
\begin{aligned}
& \frac{1}{2} \frac{d}{d t} \int\left(\rho u^{2}+|\nabla d|^{2}+\frac{1}{2}\left(|d|^{2}-1\right)^{2}\right) d x+\int\left(|\nabla u|^{2}+\left(-\Delta d+\left(|d|^{2}-1\right) d\right)^{2}\right) d x \\
& \quad \leq 0
\end{aligned}
$$

Next, we prove the following estimate:

$$
\|d\|_{L^{\infty}\left(0, T ; L^{\infty}\right)} \leq \max \left(1,\left\|d_{0}\right\|_{L^{\infty}}\right) .
$$

Without loss of generality, we assume that $1 \leq\left\|d_{0}\right\|_{L^{\infty}}$. Multiplying (1.4) by $2 d$, we get

$$
\partial_{t} \phi+u \cdot \nabla \phi-\Delta \phi+2|d|^{2} \phi=-2|d|^{2}\left(\left\|d_{0}\right\|_{L^{\infty}}^{2}-1\right)-2|\nabla d|^{2} \leq 0
$$

with $\phi:=|d|^{2}-\left\|d_{0}\right\|_{L^{\infty}}^{2}$ and $\phi(\cdot, 0)=\left|d_{0}\right|^{2}-\left\|d_{0}\right\|_{L^{\infty}}^{2} \leq 0$ and $\partial_{\nu} \phi=0$ on $\partial \Omega \times(0, \infty)$. Then (2.5) follows from (2.6) by the maximum principle.

In the following calculations, we use the following Gauss-Green formula [12]:

$$
\begin{aligned}
-\int_{\Omega} \Delta f \cdot f|f|^{p-2} d x= & \frac{1}{2} \int_{\Omega}|f|^{p-2}|\nabla f|^{2} d x+\left.\left.4 \frac{p-2}{p^{2}} \int_{\Omega}|\nabla| f\right|^{p / 2}\right|^{2} d x \\
& -\int_{\partial \Omega}|f|^{p-2}(f \cdot \nabla) f \cdot v d S-\int_{\partial \Omega}|f|^{p-2}(\operatorname{curl} f \times v) \cdot f d S
\end{aligned}
$$

and the following estimate $[13,14]$ :

$$
\|f\|_{L^{p}(\partial \Omega)} \leq C\|f\|_{L^{p}(\Omega)}^{1-\frac{1}{p}}\|f\|_{W^{1, p}(\Omega)}^{\frac{1}{p}}
$$

with $1<p<\infty$.

Taking $\nabla$ to $(1.4)_{i}$, we deduce that

$$
\partial_{t} \nabla d_{i}+(u \cdot \nabla) \nabla d_{i}+\nabla\left(\left(|d|^{2}-1\right) d_{i}\right)-\Delta \nabla d_{i}=\sum_{j} \nabla u_{j} \partial_{j} d_{i}
$$


Fan et al. Boundary Value Problems 2013, 2013:176

Page 4 of 10

http://www.boundaryvalueproblems.com/content/2013/1/176

Testing the above equation by $\left|\nabla d_{i}\right|^{p-2} \nabla d_{i}(2 \leq p \leq 6)$, using (1.1), (2.7), (2.8), (2.5) and summing over $i$, we derive

$$
\begin{aligned}
\frac{1}{p} & \frac{d}{d t} \int_{\Omega}|\nabla d|^{p} d x+\frac{1}{2} \int_{\Omega}|\nabla d|^{p-2}\left|\nabla^{2} d\right|^{2} d x+\left.\left.4 \frac{p-2}{p^{2}} \int_{\Omega}|\nabla| \nabla d\right|^{p / 2}\right|^{2} d x \\
= & -\sum_{i} \int_{\partial \Omega}\left|\nabla d_{i}\right|^{p-2}\left(\nabla d_{i} \cdot \nabla\right) v \cdot \nabla d_{i} d S+\sum_{i, j} \int_{\Omega}\left[\nabla u_{j} \partial_{j} d_{i}\left|\nabla d_{i}\right|^{p-2}\right] \cdot \nabla d_{i} d x \\
& \left.-\sum_{i} \int_{\Omega} \nabla\left(\left.|| d\right|^{2}-1\right) d_{i}\right) \cdot\left|\nabla d_{i}\right|^{p-2} \nabla d_{i} d x \\
\leq & C \int_{\partial \Omega}|\nabla d|^{p} d S-\sum_{i, j} \int_{\Omega} u_{j} \nabla \cdot\left(\partial_{j} d_{i}\left|\nabla d_{i}\right|^{p-2} \nabla d_{i}\right) d x+C \int_{\Omega}|\nabla d|^{p} d x \\
\leq & C \int_{\partial \Omega}|\nabla d|^{p} d S+\left.\left.C \int_{\Omega}|u||\nabla d|^{p / 2} \cdot|\nabla| \nabla d\right|^{p / 2}\left|d x+C \int_{\Omega}\right| \nabla d\right|^{p} d x \\
& +\left.\int_{\Omega}|u \|| \nabla d\right|^{\frac{p}{2}} \cdot|\nabla d|^{\frac{p}{2}-1}\left|\nabla^{2} d\right| d x \\
\leq & C \int_{\partial \Omega} w^{2} d S+C \int_{\Omega}|u| w|\nabla w| d x+C \int_{\Omega} w^{2} d x+\int_{\Omega}|u| w|\nabla d|^{\frac{p}{2}-1}\left|\nabla^{2} d\right| d x \\
& \left(w:=|\nabla d|^{p / 2}\right) \\
\leq & C\|w\|_{L^{2}}\|w\|_{H^{1}}+C\|u\|_{L^{q}}\|w\|{ }_{L^{\frac{2 q}{q-2}}}\|\nabla w\|_{L^{2}}+C\|w\|_{L^{2}}^{2} \\
& +C\|u\|_{L^{q}\|\nabla w\|}\left\|\frac{2 q}{{ }^{2}}\right\||\nabla d|^{\frac{p}{2}-1}\left|\nabla^{2} d\right| \|_{L^{2}} \\
\leq & 2 \frac{p-2}{p^{2}}|\nabla w|_{L^{2}}^{2}+\frac{1}{4}\left\||\nabla d|^{\frac{p}{2}-1}\left|\nabla^{2} d\right|\right\|_{L^{2}}^{2}+C\|w\|_{L^{2}}^{2}+C\|u\|_{L^{q}}^{\frac{2 q}{q-3}}\|w\|_{L^{2}}^{2}, \\
&
\end{aligned}
$$

which gives

$$
\begin{aligned}
& \frac{d}{d t} \int_{\Omega} w^{2} d x+C \int_{\Omega}|\nabla w|^{2} d x+C \int_{\Omega}|\nabla d|^{p-2}\left|\nabla^{2} d\right|^{2} d x \\
& \quad \leq C\|w\|_{L^{2}}^{2}+C\|u\|_{L^{q}}^{\frac{2 q}{q-3}}\|w\|_{L^{2}}^{2} .
\end{aligned}
$$

Therefore,

$$
\|\nabla d\|_{L^{\infty}\left(0, T ; L^{p}\right)} \leq C \quad \text { with } 2 \leq p \leq 6 .
$$

Testing (1.3) by $u_{t}$, using (1.1), (1.2), (2.1) and (2.9), we have

$$
\begin{aligned}
& \frac{1}{2} \frac{d}{d t} \int|\nabla u|^{2} d x+\int \rho\left|u_{t}\right|^{2} d x \\
& =-\int \rho u \cdot \nabla u \cdot u_{t} d x+\frac{d}{d t} \int \nabla d \odot \nabla d: \nabla u d x-2 \int \nabla d_{t} \odot \nabla d: \nabla u d x \\
& \leq\|\sqrt{\rho}\|_{L^{\infty}}\|u\|_{L^{q}}\|\nabla u\|_{L^{\frac{2 q}{q-2}}}\left\|\sqrt{\rho} u_{t}\right\|_{L^{2}} \\
& \quad+\frac{d}{d t} \int \nabla d \odot \nabla d: \nabla u d x+2\left\|\nabla d_{t}\right\|_{L^{2}}\|\nabla d\|_{L^{6}}\|\nabla u\|_{L^{3}}
\end{aligned}
$$




$$
\begin{aligned}
\leq & C\|u\|_{L^{q}}\|\nabla u\|_{L^{2}}^{1-\frac{3}{q}}\|u\|_{H^{2}}^{\frac{3}{q}}\left\|\sqrt{\rho} u_{t}\right\|_{L^{2}} \\
& +\frac{d}{d t} \int \nabla d \odot \nabla d: \nabla u d x+C\left\|\nabla d_{t}\right\|_{L^{2}}\|\nabla u\|_{L^{2}}^{1 / 2}\|u\|_{H^{2}}^{1 / 2}
\end{aligned}
$$

By the $H^{2}$-regularity theory of the Stokes system, it follows from (1.3) that

$$
\begin{aligned}
\|u\|_{H^{2}} & \leq C\left\|\nabla d^{T} \cdot \Delta d\right\|_{L^{2}}+C\left\|\rho u_{t}+\rho u \cdot \nabla u\right\|_{L^{2}} \\
& \leq C\|\nabla d\|_{L^{6}}\|\Delta d\|_{L^{3}}+C\left\|\sqrt{\rho} u_{t}\right\|_{L^{2}}+C\|u\|_{L^{q}}\|\nabla u\|_{L^{\frac{2 q}{q-2}}} \\
& \leq C\|\Delta d\|_{L^{3}}+C\left\|\sqrt{\rho} u_{t}\right\|_{L^{2}}+C\|u\|_{L^{q}}\|\nabla u\|_{L^{2}}^{1-\frac{3}{q}}\|u\|_{H^{2}}^{\frac{3}{q}} \\
& \leq \frac{1}{2}\|u\|_{H^{2}}+C\|\Delta d\|_{L^{3}}+C\left\|\sqrt{\rho} u_{t}\right\|_{L^{2}}+C\|u\|_{L^{q}}^{\frac{q}{q-3}}\|\nabla u\|_{L^{2}},
\end{aligned}
$$

which yields

$$
\|u\|_{H^{2}} \leq C\left\|\sqrt{\rho} u_{t}\right\|_{L^{2}}+C\|u\|_{L^{q}}^{\frac{q}{q-3}}\|\nabla u\|_{L^{2}}+C\|\Delta d\|_{L^{3}} .
$$

Testing (1.4) by $-\Delta d_{t}$, using (2.5) and (2.9), we obtain

$$
\begin{aligned}
& \frac{1}{2} \frac{d}{d t} \int|\Delta d|^{2} d x+\int\left|\nabla d_{t}\right|^{2} d x \\
& \quad=\int\left(\left(|d|^{2}-1\right) d+u \cdot \nabla d\right) \Delta d_{t} d x \\
& \quad \leq \int\left|\left[\nabla\left(|d|^{2} d-d\right)+\nabla(u \cdot \nabla d)\right] \nabla d_{t}\right| d x \\
& \quad \leq C\left(1+\|u\|_{L^{q}}\|\Delta d\|_{L^{\frac{2 q}{q-2}}}+\|\nabla u\|_{L^{3}}\|\nabla d\|_{L^{6}}\right)\left\|\nabla d_{t}\right\|_{L^{2}} \\
& \quad \leq C\left(1+\|u\|_{L^{q}}\|\Delta d\|_{L^{2}}^{1-\frac{3}{q}}\|d\|_{H^{3}}^{\frac{3}{q}}+\|\nabla u\|_{L^{2}}^{1 / 2}\|u\|_{H^{2}}^{1 / 2}\right)\left\|\nabla d_{t}\right\|_{L^{2}} .
\end{aligned}
$$

On the other hand, by the $H^{3}$-regularity theory of the elliptic equation, from (1.4), (2.5) and (2.9) we infer that

$$
\begin{aligned}
\|d\|_{H^{3}} & \leq C\left(\|d\|_{L^{2}}+\|\nabla \Delta d\|_{L^{2}}\right) \\
& \leq C\left(1+\left\|\nabla\left(\partial_{t} d+u \cdot \nabla d+|d|^{2} d-d\right)\right\|_{L^{2}}\right) \\
& \leq C\left(1+\left\|\nabla d_{t}\right\|_{L^{2}}+\|\nabla u\|_{L^{3}}\|\nabla d\|_{L^{6}}+\|u\|_{L^{q}}\|\Delta d\|_{L^{\frac{2 q}{q-2}}}\right) \\
& \leq C\left(1+\left\|\nabla d_{t}\right\|_{L^{2}}+\|\nabla u\|_{L^{3}}+\|u\|_{L^{q}}\|\Delta d\|_{L^{2}}^{1-\frac{3}{q}}\|d\|_{H^{3}}^{\frac{3}{q}}\right),
\end{aligned}
$$

which gives

$$
\|d\|_{H^{3}} \leq C\left(1+\left\|\nabla d_{t}\right\|_{L^{2}}+\|\nabla u\|_{L^{3}}+\|u\|_{L^{q}}^{\frac{q}{q-3}}\|\Delta d\|_{L^{2}}\right) .
$$

Combining (2.11) and (2.13), we have

$$
\begin{aligned}
\|u\|_{H^{2}}+\|d\|_{H^{3}} \leq & C+C\left\|\sqrt{\rho} u_{t}\right\|_{L^{2}}+C\left\|\nabla d_{t}\right\|_{L^{2}}+C\|\nabla u\|_{L^{2}} \\
& +C\|u\|_{L^{q}}^{\frac{q}{q-3}}\|\nabla u\|_{L^{2}}+C\|\Delta d\|_{L^{2}}+C\|u\|_{L^{q}}^{\frac{q}{q-3}}\|\Delta d\|_{L^{2}} .
\end{aligned}
$$


Putting (2.14) into (2.10) and (2.12) and summing up, we arrive at

$$
\begin{aligned}
\frac{1}{2} & \frac{d}{d t} \int\left(|\nabla u|^{2}+|\Delta d|^{2}\right) d x+\int\left(\rho\left|u_{t}\right|^{2}+\left|\nabla d_{t}\right|^{2}\right) d x-\frac{d}{d t} \int \nabla d \odot \nabla d: \nabla u d x \\
\leq & \frac{1}{4} \int \rho\left|u_{t}\right|^{2} d x+\frac{1}{4} \int\left|\nabla d_{t}\right|^{2} d x+C+C\|\nabla u\|_{L^{2}}^{2} \\
& +C\|u\|_{L^{q}}^{\frac{2 q}{q-3}}\|\nabla u\|_{L^{2}}^{2}+C\|\Delta d\|_{L^{2}}^{2}+C\|u\|_{L^{q}}^{\frac{2 q}{q-3}}\|\Delta d\|_{L^{2}}^{2},
\end{aligned}
$$

which leads to

$$
\begin{aligned}
& \|u\|_{L^{\infty}\left(0, T ; H^{1}\right)} \leq C, \quad\left\|\sqrt{\rho} u_{t}\right\|_{L^{2}\left(0, T ; L^{2}\right)} \leq C, \\
& \|d\|_{L^{\infty}\left(0, T ; H^{2}\right)}+\left\|d_{t}\right\|_{L^{2}\left(0, T ; H^{1}\right)} \leq C .
\end{aligned}
$$

It follows from (2.14), (2.15) and (2.16) that

$$
\|u\|_{L^{2}\left(0, T ; H^{2}\right)}+\|d\|_{L^{2}\left(0, T ; H^{3}\right)} \leq C .
$$

Taking $\partial_{t}$ to (1.3), testing by $u_{t}$, using (1.1), (1.2) and (2.15), we have

$$
\begin{aligned}
& \frac{1}{2} \frac{d}{d t} \int \rho\left|u_{t}\right|^{2} d x+\int\left|\nabla u_{t}\right|^{2} d x \\
& \leq\left|\int \rho u \cdot \nabla\left(u_{t}^{2}+u \cdot \nabla u \cdot u_{t}\right) d x\right|+\left|\int \rho u_{t} \cdot \nabla u \cdot u_{t} d x\right| \\
& \quad+2\left|\int \nabla d_{t} \odot \nabla d: \nabla u_{t} d x\right| \\
& \leq C\left\|\sqrt{\rho} u_{t}\right\|_{L^{2}}\|u\|_{L^{\infty}}\left\|\nabla u_{t}\right\|_{L^{2}}+C\|u\|_{L^{6}}^{2}\|\nabla u\|_{L^{6}}\left\|\nabla u_{t}\right\|_{L^{2}} \\
& \quad+C\|u\|_{L^{6}}^{2}\|\Delta u\|_{L^{2}}\left\|u_{t}\right\|_{L^{6}}+C\left\|\sqrt{\rho} u_{t}\right\|_{L^{2}}\|u\|_{L^{6}}\|\nabla u\|_{L^{6}}^{2} \\
& \quad+C\left\|\sqrt{\rho} u_{t}\right\|_{L^{2}}\left\|u_{t}\right\|_{L^{6}}\|\nabla u\|_{L^{3}}+C\left\|\nabla d_{t}\right\|_{L^{2}}\|\nabla d\|_{L^{\infty}}\left\|\nabla u_{t}\right\|_{L^{2}} \\
& \leq \frac{1}{4}\left\|\nabla u_{t}\right\|_{L^{2}}^{2}+C\|u\|_{L^{\infty}}\left\|\sqrt{\rho} u_{t}\right\|_{L^{2}}^{2}+C\|u\|_{H^{2}}^{2}+C\|u\|_{H^{2}}^{2}\left\|\sqrt{\rho} u_{t}\right\|_{L^{2}}^{2} \\
&+C\|\nabla d\|_{L^{\infty}}^{2}\left\|\nabla d_{t}\right\|_{L^{2}}^{2} .
\end{aligned}
$$

Taking $\partial_{t}$ to (1.4), testing by $-\Delta d_{t}$, using (2.5), (2.15), (2.16) and (2.17), we arrive at

$$
\begin{aligned}
\frac{1}{2} \frac{d}{d t} \int\left|\nabla d_{t}\right|^{2} d x+\int\left|\Delta d_{t}\right|^{2} d x \\
=\int\left(u \cdot \nabla d+|d|^{2} d-d\right)_{t} \cdot \Delta d_{t} d x \\
\leq \int\left[u_{t} \cdot \nabla d+u \cdot \nabla d_{t}+\left(|d|^{2} d-d\right)_{t}\right] \Delta d_{t} d x \\
=-\int \nabla\left(u_{t} \cdot \nabla d\right) \cdot \nabla d_{t} d x+\int\left[u \cdot \nabla d_{t}+\left(|d|^{2} d-d\right)_{t}\right] \Delta d_{t} d x \\
\leq\left(\left\|\nabla u_{t}\right\|_{L^{2}}\|\nabla d\|_{L^{\infty}}+\left\|u_{t}\right\|_{L^{6}}\|\Delta d\|_{L^{3}}\right)\left\|\nabla d_{t}\right\|_{L^{2}} \\
\quad+\|u\|_{L^{6}}\left\|\nabla d_{t}\right\|_{L^{3}}\left\|\Delta d_{t}\right\|_{L^{2}}+C\left\|\nabla d_{t}\right\|_{L^{2}}^{2}
\end{aligned}
$$




$$
\begin{aligned}
\leq & \frac{1}{4}\left\|\nabla u_{t}\right\|_{L^{2}}^{2}+\frac{1}{4}\left\|\Delta d_{t}\right\|_{L^{2}}^{2}+C\|\nabla d\|_{L^{\infty}}^{2}\left\|\nabla d_{t}\right\|_{L^{2}}^{2}+C\|\Delta d\|_{L^{3}}^{2}\left\|\nabla d_{t}\right\|_{L^{2}}^{2} \\
& +C\left\|\nabla d_{t}\right\|_{L^{2}}^{2}
\end{aligned}
$$

Combining (2.18) and (2.19), we have

$$
\begin{aligned}
& \left\|\sqrt{\rho} u_{t}\right\|_{L^{\infty}\left(0, T ; L^{2}\right)}+\left\|u_{t}\right\|_{L^{2}\left(0, T ; H^{1}\right)} \leq C, \\
& \left\|d_{t}\right\|_{L^{\infty}\left(0, T ; H^{1}\right)}+\left\|d_{t}\right\|_{L^{2}\left(0, T ; H^{2}\right)} \leq C .
\end{aligned}
$$

It follows from (1.4), (2.21) and (2.16) that

$$
\|d\|_{L^{\infty}\left(0, T ; H^{2}\right)} \leq C .
$$

It follows from (2.14), (2.15), (2.20) and (2.21) that

$$
\|u\|_{L^{\infty}\left(0, T ; H^{2}\right)}+\|d\|_{L^{\infty}\left(0, T ; H^{3}\right)} \leq C .
$$

It follows from (1.3), (2.20) and (2.23) that

$$
\|u\|_{L^{2}\left(0, T ; W^{2,6}\right)} \leq C,
$$

from which it follows that

$$
\|\nabla u\|_{L^{2}\left(0, T ; L^{\infty}\right)} \leq C .
$$

Applying $\nabla$ to (1.2), testing by $|\nabla \rho|^{q-2} \nabla \rho(2 \leq q \leq 6)$ and using (2.25), we have

$$
\frac{d}{d t}\|\nabla \rho\|_{L^{q}}^{q} \leq C\|\nabla u\|_{L^{\infty}}\|\nabla \rho\|_{L^{q}}^{q},
$$

which implies

$$
\|\nabla \rho\|_{L^{\infty}\left(0, T ; L^{q}\right)} \leq C,
$$

and therefore

$$
\begin{aligned}
\left\|\rho_{t}\right\|_{L^{\infty}\left(0, T ; L^{q}\right)} & =\|u \nabla \rho\|_{L^{\infty}\left(0, T ; L^{q}\right)} \\
& \leq\|u\|_{L^{\infty}\left(0, T ; L^{\infty}\right)}\|\nabla \rho\|_{L^{\infty}\left(0, T ; L^{q}\right)} \\
& \leq C .
\end{aligned}
$$

This completes the proof.

\section{Proof of Theorem 1.2}

This section is devoted to the proof of Theorem 1.2. We only need to establish a priori estimates.

First, we still have (2.1) and (2.2). 
Next, we easily infer that

$$
|d| \equiv 1 \quad \text { in }(0, \infty) \times \Omega
$$

Testing (1.9) by $-\Delta d-|\nabla d|^{2} d$ and using (1.1) and (3.1), we find that

$$
\frac{1}{2} \frac{d}{d t} \int|\nabla d|^{2} d x+\left.\left.\int|\Delta d+| \nabla d\right|^{2} d\right|^{2} d x=\int(u \cdot \nabla) d \cdot \Delta d d x
$$

Summing up (2.2) and (3.2), we have the well-known energy inequality

$$
\frac{1}{2} \frac{d}{d t} \int\left(\rho u^{2}+|\nabla d|^{2}\right) d x+\int\left(|\nabla u|^{2}+\left.\left.|\Delta d+| \nabla d\right|^{2} d\right|^{2}\right) d x \leq 0
$$

Taking $\nabla$ to $(1.9)_{i}$, testing by $\left|\nabla d_{i}\right|^{p-2} \nabla d_{i}(2 \leq p \leq 6)$, using (1.1), (2.7), (2.8) and (3.1), similarly to (2.9), we deduce that

$$
\begin{aligned}
& \frac{1}{p} \frac{d}{d t} \int_{\Omega}|\nabla d|^{p} d x+\frac{1}{2} \int_{\Omega}|\nabla d|^{p-2}\left|\nabla^{2} d\right|^{2} d x+\left.\left.4 \frac{p-2}{p^{2}} \int_{\Omega}|\nabla| \nabla d\right|^{p / 2}\right|^{2} d x \\
& =-\sum_{i} \int_{\partial \Omega}\left|\nabla d_{i}\right|^{p-2}\left(\nabla d_{i} \cdot \nabla\right) v \cdot \nabla d_{i} d S+\sum_{i, j} \int_{\Omega} \nabla u_{j} \partial_{j} d_{i}\left|\nabla d_{i}\right|^{p-2} \nabla d_{i} d x \\
& +\int_{\Omega} \nabla\left(|\nabla d|^{2} d\right) \cdot|\nabla d|^{p-2} \nabla d d x \\
& \leq \frac{p-2}{p^{2}} \int_{\Omega}|\nabla w|^{2} d x+C\|w\|_{L^{2}}^{2}+C\|u\|_{L^{q}}^{\frac{2 q}{q-3}}\|w\|_{L^{2}}^{2} \\
& +\int_{\Omega}|\nabla d|^{2} w^{2} d x+C \int_{\Omega}|\nabla d|\left|\nabla^{2} d\right||\nabla d|^{\frac{p}{2}-1}|\nabla d|^{\frac{p}{2}} d x \quad\left(w:=|\nabla d|^{p / 2}\right) \\
& \leq \frac{p-2}{p^{2}} \int_{\Omega}|\nabla w|^{2} d x+C\|w\|_{L^{2}}^{2}+C\|u\|_{L^{q}}^{\frac{2 q}{q-3}}\|w\|_{L^{2}}^{2} \\
& +\|\nabla d\|_{L^{q}}^{2}\|w\|_{L^{\frac{2 q}{q-2}}}^{2}+\frac{1}{4} \int_{\Omega}|\nabla d|^{p-2}\left|\nabla^{2} d\right|^{2} d x \\
& \leq 2 \frac{p-2}{p^{2}} \int_{\Omega}|\nabla w|^{2} d x+C\|w\|_{L^{2}}^{2}+C\|u\|_{L^{q}}^{\frac{2 q}{q-3}}\|w\|_{L^{2}}^{2} \\
& +C\|\nabla d\|_{L^{q}}^{\frac{2 q}{q-3}}\|w\|_{L^{2}}^{2}+\frac{1}{4} \int_{\Omega}|\nabla d|^{p-2}\left|\nabla^{2} d\right|^{2} d x,
\end{aligned}
$$

which yields

$$
\|\nabla d\|_{L^{\infty}\left(0, T ; L^{p}\right)}+\int_{0}^{T} \int|\nabla d|^{2}\left|\nabla^{2} d\right|^{2} d x d t \leq C .
$$

We still have (2.10) and (2.11).

Similarly to (2.12), testing (1.9) by $-\Delta d_{t}$, using (3.1) and (3.4), we get

$$
\begin{aligned}
\frac{1}{2} & \frac{d}{d t} \int|\Delta d|^{2} d x+\int\left|\nabla d_{t}\right|^{2} d x \\
\quad & =\int\left(u \cdot \nabla d-|\nabla d|^{2} d\right) \Delta d_{t} d x
\end{aligned}
$$




$$
\begin{aligned}
= & \int_{\Omega} \nabla\left(|\nabla d|^{2} d-u \cdot \nabla d\right) \cdot \nabla d_{t} d x \\
\leq & C\left(1+\|u\|_{L^{q}}\|\Delta d\|_{L^{\frac{2 q}{q-2}}}+\|\nabla u\|_{L^{3}}\|\nabla d\|_{L^{6}}\right)\left\|\nabla d_{t}\right\|_{L^{2}} \\
& +C\left(\|\nabla d\|_{L^{6}}^{3}+\left\|\nabla|\nabla d|^{2}\right\|_{L^{2}}\right)\left\|\nabla d_{t}\right\|_{L^{2}} \\
\leq & C\left(1+\|u\|_{L^{q}}\|\Delta d\|_{L^{2}}^{1-\frac{3}{q}}\|d\|_{H^{3}}^{\frac{3}{q}}+\|\nabla u\|_{L^{2}}^{1 / 2}\|u\|_{H^{2}}^{1 / 2}+\left\|\nabla|\nabla d|^{2}\right\|_{L^{2}}\right)\left\|\nabla d_{t}\right\|_{L^{2}} .
\end{aligned}
$$

Similarly to (2.13), we have

$$
\begin{aligned}
\|d\|_{H^{3}} & \leq C\left(1+\left\|\nabla d_{t}\right\|_{L^{2}}+\|\nabla u\|_{L^{3}}+\|u\|_{L^{q}}^{\frac{q}{q-3}}\|\Delta d\|_{L^{2}}+\left\|\nabla\left(|\nabla d|^{2} d\right)\right\|_{L^{2}}\right) \\
& \leq C\left(1+\left\|\nabla d_{t}\right\|_{L^{2}}+\|\nabla u\|_{L^{3}}+\|u\|_{L^{q}}^{\frac{q}{q-3}}\|\Delta d\|_{L^{2}}+\left\|\nabla|\nabla d|^{2}\right\|_{L^{2}}\right) .
\end{aligned}
$$

Combining (2.11) and (3.6), we have

$$
\|u\|_{H^{2}}+\|d\|_{H^{3}} \leq \text { the right hand side of (2.14) }+C\left\|\nabla|\nabla d|^{2}\right\|_{L^{2}} \text {. }
$$

Putting (3.7) into (3.5) and (2.10) and using the Gronwall inequality, we still have (2.15), (2.16), (2.17) and (2.18).

Similarly to (2.19), applying $\partial_{t}$ to (1.9), testing by $-\Delta d_{t}$ and using (3.4), we have

$$
\begin{aligned}
\frac{1}{2} & \frac{d}{d t} \int\left|\nabla d_{t}\right|^{2} d x+\int\left|\Delta d_{t}\right|^{2} d x \\
= & \int\left(u \cdot \nabla d-|\nabla d|^{2} d\right)_{t} \cdot \Delta d_{t} d x \\
= & \int\left(u_{t} \cdot \nabla d+u \cdot \nabla d_{t}-|\nabla d|^{2} d_{t}-d \partial_{t}|\nabla d|^{2}\right) \Delta d_{t} d x \\
= & -\int \nabla\left(u_{t} \cdot \nabla d\right) \cdot \nabla d_{t} d x+\int\left(u \cdot \nabla d_{t}-|\nabla d|^{2} d_{t}-d \partial_{t}|\nabla d|^{2}\right) \Delta d_{t} d x \\
\leq & \frac{1}{4}\left\|\nabla u_{t}\right\|_{L^{2}}^{2}+\frac{1}{4}\left\|\Delta d_{t}\right\|_{L^{2}}^{2}+C\|\nabla d\|_{L^{\infty}}^{2}\left\|\nabla d_{t}\right\|_{L^{2}}^{2} \\
& +C\|\Delta d\|_{L^{3}}^{2}\left\|\nabla d_{t}\right\|_{L^{2}}^{2}+C\left\|\nabla d_{t}\right\|_{L^{2}}^{2} .
\end{aligned}
$$

Combining (2.18) and (3.8) and using the Gronwall inequality, we still obtain (2.20) and (2.21).

By similar calculations as those in (2.22)-(2.27), we still arrive at (2.22)-(2.27).

This completes the proof.

Competing interests

The authors declare that they have no competing interests.

\section{Authors' contributions}

All authors read and approved the final manuscript.

\section{Author details}

'Department of Applied Mathematics, Nanjing Forestry University, Nanjing, P.R. China. ${ }^{2}$ Department of Mathematics, Inha University, Incheon, 402-751, Republic of Korea. ${ }^{3}$ Department of Mathematics, Zhejiang Normal University, Jinhua, P.R. China. 


\section{Acknowledgements}

This work is partially supported by the Zhejiang Innovation Project (Grant No. T200905), the ZJNSF (Grant No. R6090109) and the NSFC (Grant No. 11171154). The authors are indebted to the referee for some helpful suggestions.

Received: 9 April 2013 Accepted: 11 July 2013 Published: 26 July 2013

\section{References}

1. Chandrasekhar, S: Liquid Crystals, 2nd edn. Cambridge University Press, Cambridge (1992)

2. Ericksen, JL: Hydrostatic theory of liquid crystals. Arch. Ration. Mech. Anal. 9, 371-378 (1962)

3. Leslie, FM: Some constitutive equations for liquid crystals. Arch. Ration. Mech. Anal. 28(4), 265-283 (1968)

4. Lin, F-H, Liu, C: Nonparabolic dissipative systems modeling the flow of liquid crystals. Commun. Pure Appl. Math. 48(5), 501-537 (1995)

5. Danchin, R: Density-dependent incompressible fluids in bounded domains. J. Math. Fluid Mech. 8(3), $333-381$ (2006)

6. Kim, H: A blow-up criterion for the nonhomogeneous incompressible Navier-Stokes equations. SIAM J. Math. Anal. 37(5), 1417-1434 (2006)

7. Fan, J, Ozawa, T: Regularity criteria for the 3D density-dependent Boussinesq equations. Nonlinearity 22(3), 553-568 (2009)

8. Guillén-González, F, Rojas-Medar, MA, Rodríguez-Bellido, MA: Sufficient conditions for regularity and uniqueness of a 3D nematic liquid crystal model. Math. Nachr. 282(6), 846-867 (2009)

9. Li, X, Wang, D: Global strong solution to the density-dependent incompressible flow of liquid crystals. arXiv:1202.1011v1 [math. AP] 5 Feb 2012

10. Huang, $X$, Wang, Y: Global strong solution to the $2 \mathrm{D}$ nonhomogeneous incompressible MHD system. J. Differ. Equ. 254(2), 511-527 (2013)

11. Fan, J, Gao, H, Guo, B: Regularity criteria for the Navier-Stokes-Landau-Lifshitz system. J. Math. Anal. Appl. 363(1), 29-37 (2010)

12. Beirão da Veiga, $H$, Crispo, F: Sharp inviscid limit results under Navier type boundary conditions. An $L^{p}$ theory. J. Math. Fluid Mech. 12, 397-411 (2010)

13. Adams, RA, Fournier, JF: Sobolev Spaces, 2nd edn. Pure and Appl. Math., vol. 140. Elsevier, Amsterdam (2003)

14. Lunardi, A: Interpolation Theory, 2nd edn. Lecture Notes. Scuola Normale Superiore di Pisa (New Series). Edizioni della Normale, Pisa (2009)

doi:10.1186/1687-2770-2013-176

Cite this article as: Fan et al.: Blow-up criteria for 3D nematic liquid crystal models in a bounded domain. Boundary Value Problems 2013 2013:176.

\section{Submit your manuscript to a SpringerOpen ${ }^{\circ}$ journal and benefit from:}

- Convenient online submission

- Rigorous peer review

Immediate publication on acceptance

- Open access: articles freely available online

- High visibility within the field

- Retaining the copyright to your article 